\title{
El profesorado y "su lugar" para estudiantes de Bachillerato. Hallazgos de una investigación sobre trayectorias de éxito escolar
}

The teachers and their "place" for high school students. Findings from research on academic paths of school success

\author{
Dolo Molina Galvañ* \\ Universidad de Valencia \\ Nieves Blanco** \\ Universidad de Málaga \\ Soledad García*** \\ Universidad de Sevilla
}

Resumen Distintos estudios sobre el éxito escolar confirman que una relación positiva con el profesorado puede favorecer el compromiso con la escuela. Subrayan que el profesorado favorece el éxito escolar cuando aporta una combinación de apoyo y de exigencia, que se sostiene en la valoración y la confianza en las capacidades de sus estudiantes. El presente texto recupera los significados que estudiantes de Bachillerato, con trayectoria de éxito escolar, le dan a su experiencia formativa e identifica algunos de los elementos del actuar docente que ayuda a estos jóvenes a establecer una relación positiva con la escuela.

Palabras-Chave: Éxito escolar, Profesorado, Relación educativa, Enseñanza secundaria, Estudios biográficos.

Abstract Several studies about school success confirm that a positive teacherstudent relationship can favor a strong engagement to school life. Such studies emphasize the fact that teachers contribute to school success when they combine both a demanding and a supportive attitude towards their students, such attitude arising from a sense of value and trust in their students' capabilities. This paper examines the different meaning that successful secondary education students give to their academic experience and defines some of the specific features of teaching which help to create a positive school-student relationship.

KEYWORDS: School success, Teachers, Teacher-student Relationship, Secondary education, Biographical studies. 


\section{A modo de introducción}

El resultado de un rendimiento no es el resultado de un chico o de una chica, es el resultado de la calidad de la relación que hay entre ella/él y la/el enseñante. Vita Cosentino (2010, p. 141)

Los hallazgos que presentamos forman parte de una investigación cuyo foco de estudio es el éxito escolar. En particular, tratamos de conocer qué factores pedagógicos favorecen el éxito escolar en jóvenes de enseñanza secundaria postobligatoria. ${ }^{1}$ En el estudio, desarrollado desde una perspectiva biográfica, se concibe el éxito como proceso que se va construyendo en una trayectoria que puede iniciarse, incluso, tras un paso difícil por la escolaridad. Este modo de dar sentido al éxito sugiere que la relación que se establece con la escuela es siempre una relación viva, que se crea y recrea através de las mediaciones y que cada estudiante la experimenta de manera singular. Por ello, cuando hablamos de estudiantes de Bachillerato con trayectoria de éxito escolar nos referimos a jóvenes que han establecido una relación positiva con la escuela alcanzando los estándares exigidos; jóvenes que han mantenido una continuidad en los estudios (superando, en ocasiones, dificultades sociales y personales) y que han encontrado, además, una manera de ir a la escuela susceptible de encontrar placer.

Este texto recoge algunas de las reflexiones que hemos ido abriendo al preguntarnos acerca del "lugar" que ocupa el profesorado en las trayectorias de éxito de estas y estos estudiantes. Lugar reconocible cuando la presencia, la relación y la actuación del profesorado ha afectado de alguna forma al estudiante. Es decir, cuando se ha experimentando un vínculo en el que ha estado presente el reconocimiento y se ha dado, además, sentido a esos tiempos y espacios educativos en los que actúa la vía de transmisión. Así, preguntarnos sobre el lugar que ocupa el profesorado nos lleva a colocarnos en la lógica de la mediación ${ }^{2}$ y no del orden; pues se interroga la relación educativa en cuanto relación con la alteridad (las y los estudiantes) y en cuanto relación que se establece (y se ayuda a establecer) con el saber.

Nos ha preocupado, a la hora de interpretar qué papel juega el profesorado como factor favorecedor del éxito escolar, colocar la mirada en la relación educativa. Y hacerlo sin quedar fijadas en las preguntas "sobre el qué" y "sobre el cómo", es decir, sobre los contenidos o las didácticas; pues partiendo de la experiencia de las y los estudiantes lo que tratamos de desvelar es cómo han ido viviendo la escuela y cómo han ido dando un sentido propio a su estar y hacer en la escuela. 


\section{A propósito del éxito escolar. Situar el objeto de la investigación}

Para que el vínculo educativo se sostenga debe producirse el consentimiento del sujeto. Hebe Tizio (2007, p. 196)

La desafección de los y las jóvenes hacia la escuela es una de las grandes preocupaciones sociales. Teniendo en cuenta que el progreso social, en las sociedades de la información, depende cada vez más de la formación de sus ciudadanas y ciudadanos, el interés por lograr mayores tasas de éxito durante la escolaridad obligatoria y post-obligatoria, se ha convertido en uno de los principales objetivos de la política internacional y nacional. La Unión Europea adopta como objetivo prioritario para el 2020, que el porcentaje de jóvenes entre 18-24 años que abandona de forma temprana la educación y la formación no supere $10 \%$. Un gran reto, considerando que las tasas de abandono escolar son del 32\% para España y del $38 \%$ para Andalucía (contexto de este estudio).

Los informes nacionales e internacionales confirman que es insostenible el actual nivel de abandono e insatisfacción de los jóvenes hacia la escuela. En términos educativos, el problema no se resuelve únicamente desde el propósito de retener cada vez a más jóvenes mayores de 16 años dentro del sistema formativo; es necesario además, que transiten por él con éxito. Por ello, teniendo en cuenta que en nuestro sistema educativo también hay estudiantes que tienen éxito, es decir, que completan las etapas educativas, que se gradúan, que se forman en el sentido más integrador y amplio del término; un modo posible para avanzar en abordajes e intervenciones más globales del problema sea colocar aquí la mirada. Si lo que pretendemos es intervenir para logar mayores niveles de continuidad y éxito en la enseñanza, necesitamos identificar las condiciones que favorecen estas trayectorias de éxito. Se trata de desplazar la mirada, algo que nos parece especialmente relevante, para reorientar las prácticas educativas en los primeros niveles educativos, que es donde comienzan a gestarse las condiciones que llevan al abandono o a la continuidad de la formación en nuestros sistemas educativos.

Este estudio trata pues, de significar y visibilizar el éxito escolar en la educación secundaria, de analizar las experiencias y trayectorias de estudiantes que dan continuidad a su vida escolar con éxito académico. En esta tarea, la primera constatación que tenemos es que el concepto de éxito escolar se utiliza con excesiva simplicidad y bastante ambigüedad; definiéndolo como reducción del abandono escolar o asociándolo a las altas calificaciones o identificándolo desde el logro de credenciales obtenidas. Se advierte pues, una perspectiva dominante centrada en la lógica de constatación de los resultados y la rendición de cuentas, algo que no resulta extraño desde la entrada en escena del informe PISA y la utilización mediática y política del mismo (HERNÁNDEZ; TORT, 2009). El problema es que esta lógica intenta hacer semejantes y por tanto intercambiables, las finalidades educativas con los resultados. Haciendo crecer, a su vez, una mirada valorativa sobre las instituciones educativas basada en la lógica de las carencias: lo que no hace la escuela, lo que no consiguen los estudiantes, lo que no logra la educación, lo que le falta al profesorado. 
Sabemos, sin embargo, que el éxito escolar es un constructo complejo, con múltiples dimensiones en las que se entrelazan aspectos biográficos-personales y aspectos socio-históricos e institucionales. Estudiar el éxito escolar más allá del referente de la rendición de cuentas, nos parece una tarea necesaria y urgente. Y trabajar este constructo desde una perspectiva biográfica permite atender mejor a la complejidad del proceso así como tener referentes para valorar los efectos, y no tanto los resultados, que produce la escuela. Es decir, para desplazar una política basada únicamente en la gestión y dar cabida a una política humana y cultural más acorde con la lógica formativa de nuestra instituciones educativas. Este es el sentido que orienta nuestro trabajo. Los datos que presentamos proceden de entrevistas semiestructuradas a una muestra intencional de 16 estudiantes de Bachillerato (7 chicas y 9 chicos); y se han completado con la técnica de foto-lenguaje para explorar cuestiones sobre su trayectoria escolar, y un cuestionario con información sobre su contexto sociofamiliar. Además de las entrevistas mantenidas con las y los estudiantes, hemos realizado entrevistas a algunas y algunos docentes que estos estudiantes han identificado como referentes significativos en su trayectoria escolar. $^{3}$

\section{La presencia del profesorado en la experiencia de éxito escolar}

Sus explicaciones estaban siempre un poco más arriba de mi cabeza, así que mi cerebro tenía que estar de puntillas la mayor parte del tiempo. Elizabeth Ellsworth (2005, p. 13)

Por el carácter relacional de la educación sabemos que el profesorado tiene un papel básico en la experiencia formativa de las y los estudiantes. Así, las investigaciones sobre éxito escolar, destacan la importancia crucial del profesorado para generar experiencias positivas de relación con la escuela. En ellas se subraya que el profesorado favorece el éxito escolar cuando aporta una combinación de apoyo y exigencia que se sostiene en la valoración y la confianza en las capacidades y posibilidades de sus estudiantes. Favorece el éxito escolar cuando, además, esta combinación de apoyo y exigencia está acompañada de la competencia académica del profesorado. De manera que, confiar en la capacidad de sus estudiantes, apoyar los logros académicos y dar sentido a los saberes que se ponen a disposición de las y los estudiantes; son tres claves relacionadas con el hacer docente que los estudios realizados apuntan como favorecedoras del éxito escolar.

Considerando estas aportaciones focalizamos nuestra pregunta en la práctica de la mediación educativa. Es decir, en el actuar docente. Nos preocupa explorar y buscar los significados que estos jóvenes dan al actuar docente que trata de poner en relación a las y los estudiantes con el saber. Nos preocupa porque el sentido que dan a su estar y hacer en la escuela no se encuentra al margen de la relación que establecen con el saber. También, porque es a través de estos saberes, que el profesorado tiene la posibilidad de relacionarse con las experiencias y las expectativas de las y los jóvenes, (BLANCO, 2006). Se trata pues, de una mediación educativa que pone de relieve dos dimensiones fundamentales en la escuela: los saberes y las relaciones. 
Un primer acercamiento a los relatos de estas y estos estudiantes nos sitúa ante el hecho de reconocer que si bien el profesorado está presente en su experiencia formativa, no siempre adquiere presencia. Esta diferencia entre "estar presente" y "tener presencia" 4 se evidencia muy bien en los relatos analizados. Considerando que la presencia se reconoce al reconocer las implicaciones transformadoras que el actuar docente provoca en cada quién, desde el punto de vista de las y los estudiantes, el profesorado que deja huella es aquel a quien especialmente reconocen por su labor. ¿Cómo van identificando, nombrando y significado esta mediación educativa?

A su vez, en este acercamiento a los relatos, se constata que las chicas han descrito con más detenimiento, intensidad y detalle su experiencia formativa. Han hablado más y han aportado mayor cantidad de información sobre la naturaleza de los vínculos; han mostrado bastante más facilidad que los chicos para hablar y pensar sobre sí mismas, para matizar y para reparar en los aspectos más sutiles de la mediación educativa. Una segunda constatación es que los relatos han ido organizándose alrededor de dos grandes planos de su experiencia formativa: la relación con la materia y el trabajo en el aula, y la relación con el profesorado. Y es a partir de estos hilos organizadores que interpretamos y ordenamos sus palabras.

\section{Saber y saber transmitir: la relación con la materia}

Destacan con insistencia la importancia de que el profesorado sepa transmitir lo que sabe. Que prepare las clases y dedique tiempo a explicar y asegurarse que se comprende todo lo que se está explicando, bien sea un concepto, o una pregunta o una tarea..$^{5}$ Saber transmitir, para estas y estos estudiantes, coincide con el "saber explicar". Es decir, que las explicaciones sean claras, que intenten desenredar la complejidad sin simplificar lo que se dice. A veces hay que repetir lo ya dicho, pero se trata también de no acelerar el ritmo ni de sobrepasar dificultades. Valoran que el saber se vincule con algo que puedan reconocer. "Me quedo con la clases de sociales del año pasado. La historia la hacía no sólo interesante, sino que la entendieras. [...] Los demás te dicen que ha pasado esto y esto y tú lo aprendes de memoria; pero claro si te dicen los motivos lo aprendes mejor", (Diego).

Saber explicar no está separado del saber. Tener certeza de que el profesorado sabe de lo que habla es importante para estas y estos estudiantes. "Me gustan los profesores que sepan”, dice Jaime. Les da tranquilidad y confianza, sobre todo, cierta seguridad de que van a aprender y esto parece más importante que la propia materia. "Si el profesor se desenvuelve bien, no me va a importar demasiado la materia", (Pablo). Pero cuando insisten en que el profesorado sepa, se refieren en un sentido más amplio a la competencia académica. A veces, dicha competencia se reconoce como dedicación al trabajo. "No es profesora durante seis horas que está aquí sino que ella sabe muchísimo y aprende todos los días y hace que te guste la asignatura”, (Lola). Otras veces, se asocia con el valor que se le da al conocimiento, al hecho de saber para comprender, más allá del dominio de una materia concreta. "Veía que el profesor tenía varias carreras: Historia, Derecho, 
Economía [me ayudó a ver] que no tienes que cerrarte a ningún campo", (David). O de otro modo, insiste Lola, tener dominio de lo que se sabe es importante para ayudar al alumnado a establecer una relación positiva con el saber: "Yo no tengo buena memoria [...] y temía muchísimo a la historia. No me gustaba. Pero con la profesora que tengo es que [vas a clase] te cuenta un cuento y dices me encanta. Ahora es una de mis asignaturas preferidas".

Son firmes al asegurar que estas cualidades que buscan en el profesorado de "saber" y "saber explicar" son reconocibles a través del tacto, de la sensibilidad, de la disponibilidad. Hablan de un profesorado que transmite alegría. "Ese hombre me transmitía alegría. Muy dinámico. Nos hacía la historia que no era un tocho, y eso que los exámenes eran dificilillos”, (Diego). Hablan, también, de cercanía, de actividad, de acompañar, de hacer lugar. "En mi colegio, además de enseñarte cosas [...] hacíamos muchas cosas. Te divertías muchísimo. Hacían que vinieras al colegio con ganas de estar en el colegio. No venías al colegio diciendo otro día más”, (Susi). Y sobre todo, hacen presente la pasión. La pasión por lo que se enseña que el alumnado vive como compromiso con la verdad de lo que se transmite. "[Valoro] que le guste de verdad su asignatura, que se vea que le apasiona. [...]Tuve una profesora que daba lengua y decía qué bonito es leer, o profesores de biología y geología que están locos por las piedras. La geología en sí no es bonita; son piedras, pues no sé como consiguió que nos encantasen las piedras, nos llevaba al laboratorio y se emocionaba", (Lola).

Es interesante además, atender a la reflexión con la que estas estudiantes matizan el sentido y significado de esta mediación educativa. De este actuar docente que les pone en relación con el saber. Señalan, por ejemplo, que saber mucho no significa saberlo todo. "Eso es lo que más me gusta de ella, no piensa que lo sabe todo, sino que siempre va aprendiendo", (Lola). En realidad, no exigen al profesorado que lo sepa todo ni que tenga todas las respuestas. Se trata mas bien, de tener confianza en que lo imprevisto tiene cabida en el trabajo de aula. "Si se olvida de algo, sabes que lo sabe, que no es alguien que te dice eso es así y ya está y te lo tienes que aprender de esta manera", (Lola). Ni se trata de repetir por repetir ni de memorizar por memorizar. Lo que se valora, lo que se busca en el profesorado, es ese saber dar vida a aquello que se enseña. "Ella lo que hace es explicárnoslo de tal forma que las cosas se sitúan en un contexto, no tienes que aprendértelas de memoria. Sabiendo de donde vienen las cosas, entiendes hacia donde van", (Sara). Y se refieren a los detalles, al gusto por los detalles, que muestra el profesorado que trata de vivificar el saber. "Te habla de un edificio y no sólo es que te vaya a hablar del edificio sino de las familias que había en el edificio. Te dice el nombre de las familias, todos los detalles", (Lola). La referencia a los detalles aquí, señala una diferencia respecto de buscar ejemplos prácticos que clarifican una idea, un concepto, un enunciado. Vivificar, dar vida, es algo diferente a buscar y traer "traducciones" prácticas de la teoría. 


\section{Hacer reconocible la disparidad: la relación con el profesorado}

Un buen ejemplo de que la relación educativa no es reducible a los afectos que provocamos en nuestros estudiantes, se encuentra en esto que las y los estudiantes reclaman: no confundir la cercanía con el colegueo. Experimentan la cercanía del profesorado como un dar confianza por ejemplo, para preguntar o intervenir en clase. "Me gusta tener confianza con los profesores porque al tener más confianza puedo preguntarle más dudas. [...] Si no tuviese confianza, pues yo, por ejemplo, difícil”, (Pepe). Esta cercanía que da confianza se percibe al sentirse escuchados, al hacerse accesible, al relacionarse de una manera distendida; pero es importante, para estas y estos estudiantes, no confundirse. Es decir, no transformar la relación con el profesorado en una relación entre iguales. "Un profesor es un profesor y no tiene que superar la línea del colegueo. Pero es mejor que el profesor sea menos distante con los alumnos [...] Si es más cercano tienes como más confianza de preguntarle cosas, de decirle cosas", (Raquel). Se pide al profesorado que delimite la distancia necesaria y la proximidad posible. "El profesor debe marcar las distancias [...]. Esa distancia la tiene que marcar muy bien, pues hay quienes son buena gente, pero lo toman por tonto; y otros que son buena gente pero que saben hasta donde pueden llegar con él”, explica Diego. Aluden a la necesaria presencia de la disparidad y la alteridad en la relación educativa. ${ }^{6}$ Sin embargo, observamos que chicas y chicos lo significan de manera diferente. Ellos hablan más en términos de posiciones y funciones, "el profesor debe imponer respeto" o "el profesor debe marcar las distancias"; ellas hacen más referencia a su estar, al modo en que se sienten afectadas, "no soy capaz de estar mucho rato hablando con los profesores" o "no te da tanto miedo" o "se relaciona contigo como amiga pero sin perder las distancias de profesora".

Esperan del profesorado que tenga autoridad en el aula, algo que reconocen y saben distinguir muy bien del autoritarismo. Por ello valoran que estén disponibles para la enseñanza y cercanos y preocupados para hacerles una llamada de atención si hace falta. "Nos exigía mucho, [...] pero me gustaba porque se interesaba por nosotros", (Pepe). "Nos disciplinaba, nos preparaba para lo que había que hacer", (Jaime). Esta exigencia la viven como estímulo y motivación cuando sienten que el profesorado confía en sus posibilidades, cuando perciben que se tira de ellos. Distinguen entre exigencia y control, "que te digan estudia, eso me lo dice también mi padre", explica Sonia. La exigencia es otra cosa, se vive de otra forma; como una manera de devolver la confianza en una misma y ayudar a sacar lo mejor de cada quién. "Es la forma de concienciarte de que estudiar es bueno. No sé. Te hace ver las cosas mejor", (Sonia). Así, la seriedad y la exigencia en el trabajo que buscan en el profesorado no la contraponen al deseo de que se establezca un ambiente cordial y distendido, afectuoso, incluso.

Aseveran con naturalidad y aplomo que el trabajo docente no está destinado para cualquier persona. La enseñanza no debe ser una salida para asegurarse un sueldo; en esta elección ha de haber otras motivaciones. "Hay maestros funcionarios que no tienen deseo de docencia y hay algunos que sí. Eso es importante porque si tú quieres ser funcionario, si quieres tener un sueldo 
fijo, no sé, búscate otra rama pero es que la educación es el futuro", (David). Buscan en el profesorado que sea entusiasta, profesional, responsable, cercano porque cuando algo de esto está presente el vínculo es más vivo y más fuerte. Y en este matiz, las chicas nombran con mayor detenimiento lo que posibilita y da fuerza a este vínculo. Por ejemplo, el sentido del humor: "elegiría a mi profesor de matemáticas, las clases son muy divertidas. Es muy bromista pero cuando tiene que ponerse serio se pone", (Irene). O el ser paciente y saber acompañar: "Una profesora que estaba mucho con nosotros. Nos explicaba las cosas y se esforzaba mucho porque lo entendiéramos", (Rocío). O el saber escuchar y un "saber sentir con": "Ella sabía perfectamente cuando tenías un problema. Estar sin pasar el límite, si no quieres decir algo no se van a enfadar pero se preocupan por ti", (Lola). O el hacer presente y palpable el gusto por lo que hacen, "los profesores que les gusta los que hacen en general tienen un trato especial con los alumnos", (Lola).

Pero si este tono de amorosidad que combina cercanía y exigencia es lo que hace posible la relación educativa; aquello que la cierra y la imposibilita no es tanto la distancia como la indiferencia. "No es que haya tenido malos profesores ni nada de eso, pero no hay ningún tipo de acercamiento. Algunos, después de un año no se saben ni tu nombre", (Susi). Donde no hay vínculo no es posible el reconocimiento. No es posible la autoridad ni la relación.

\section{Algunas consideraciones finales...}

¿Qué hace falta para que algo se constituya como lugar? Básicamente, que no sea un espacio de anonimato. Eduardo Corbo (2007, p. 308)

Diferentes autores y autoras apuntan que si la escuela tiene sentido para las y los jóvenes es porque han conseguido dar un sentido propio a su estar y hacer en la escuela. Y en este dar un sentido propio lo que está presente es una experiencia de éxito escolar. Siguiendo este hilo, en nuestro estudio nos ha preocupado saber y significar cómo ha sido y cómo han vivido las y los estudiantes con trayectoria de éxito escolar su experiencia formativa. En relación con ello, lo que hemos desarrollado en este texto de manera más específica, es la pregunta sobre el lugar que ocupa el profesorado en su experiencia formativa.

La pregunta no pretendía centrarse en aquello que viene marcado como lleno, sólido o dado porque nos llevaría a cuestiones de contenidos y didácticas que, aunque hay que mantener abierta la necesidad de repensarlas y discutirlas, no son nuestro centro de interés aquí. La pregunta nos situaba ante la referencia de lo "vacío", lo "líquido" y lo "por hacer"; buscando comprender mejor la distinción entre estar presente y tener presencia. Por ello no colocamos el peso de la reflexión en la adecuación de las estrategias pedagógicas que utiliza el profesorado para llegar de forma más precisa y directa, eficiente y efectiva, a sus estudiantes. La pedagogía es un asunto más complicado, y sobre todo, no concluyente, (ELLSWORTH, 2005). Nos preocupa más bien, atender al modo 
en que se articula y se encarna la relación educativa, y hacerlo atendiendo al hecho de que no es posible en ausencia del vínculo. Si la relación educativa, además, no se conforma en un conjunto de normas ni de estrategias que pretenden modularla en esa lógica dominante de la eficacia y la efectividad; de lo que se trata es de explorar y descubrir cualidades sobre cómo generar, cuidar y sostener ese vínculo que hace posible una relación educativa fértil.

Descubrimos, en estos relatos, que el profesorado adquiere presencia no desde lo que hace (o no sólo desde aquí), sino desde su actuar. Actuar en el sentido arendtiano de tomar iniciativa, de poner algo en movimiento. Por ello, decimos que el lugar que ocupa el profesorado en la experiencia formativa de las y los estudiantes es también un lugar vacío, líquido y por hacer; porque es en este lado del "lugar" donde se despliega el actuar.

\section{Un lugar vacío}

Lo que posibilita la presencia del profesorado no es la relación que viene dada por la propia institución entre profesor/a - estudiante. Es otra cosa. Requiere que ese lugar de la relación esté vacío para que se revele un quién. Así, al reconocer experiencias formativas que han posibilitado la transformación de sí, no se nombra "lo que me gustaría" que fuera o hiciese tal o cual profesor sino aquello que aconteció en la relación viva y encarnada. Los relatos nos descubren que es el profesorado quien se arriesga a ocupar y quien torna habitable este lugar. Quien se arriesga sin transitar de cualquier manera ni llenarlo de cualquier cosa. Por ello, la relación educativa es una relación afectiva, sensible, con el otro y con el saber. Porque no es sólo el otro (estudiante) o el saber, sino la relación que procuramos con el otro y con el saber lo que concede la cualidad simbólica de la relación educativa; y lo que coloca a la educación como una forma que tenemos para modificar nuestra relación con la realidad.

\section{Un lugar líquido}

Lo que posibilita la presencia del profesorado no es el peso del sujeto (su omnipresencia) sino su descentralización. Para las y los estudiantes no sólo es importante que el profesor o la profesora sea presente en sus vidas, sino que ellos y ellas puedan tener lugar en la vida del profesor o la profesora. Para hacer este lugar, el profesorado, precisa ponerse en disposición para vivir la pluralidad y la singularidad. Precisa aprender a moverse, a navegar, entre el "todos" y el "uno" y hacerlo fuera de la lógica escolar que se basa en el supuesto de la homogeneidad. Salir de este principio organizador en lo escolar, implica explorar formas de relación que permitan al profesorado dejarse tocar por el otro, por quien tiene delante, el o la estudiante. Explorar y asumir formas de relación en las cuales está presente el conflicto de sí; pues sin eludir quien se es, lo cierto es que vamos siendo en un haz de relaciones. De manera que a veces, ir hacia el otro comporta deshacerse un poco del yo. Pero esta imagen líquida no supone la liquidación de la relación, ${ }^{7}$ apunta más bien que la relación educativa que tiene origen en el aula crece en cada quien más allá, fuera, de ella. 


\section{Un lugar por hacer}

Lo que posibilita la presencia del profesorado no es sólo lo que sabe y ha aprendido en su relación con la infancia y la juventud; lo que realmente posibilita la presencia es lo que no sabe y aquello que se atreve a desaprender para continuar acogiendo lo nuevo. Mantener abierta la interrogación sobre las necesidades y los deseos del otro. Y mantener abierta junto a ella, la vía de interrogación propia que permite tomar decisiones sensatas en cada situación, la que cada situación pensamos que requiere, confiando en el saber de nuestra experiencia. Se trata, quizás, de mantener una relación pensante con la realidad (y con la juventud) que tenemos delante, aceptando a su vez, que la realidad (y la juventud) va cambiando.

\section{Referencias}

ARENDT, H. Entre el pasado y el futuro. Barcelona: Península, 2003.

BLANCO, N. El sentido de los saberes escolares: la experiencia de escolarización de alumnas de enseñanza secundaria. In: XI Congreso Nacional de Investigación Educativa (Comie) en XI Congreso Nacional de Investigación Educativa (COMIE). 2011. México D.F.

Saber para vivir. In: PIUSSI, A. M.; MAÑERU, A. (Org.). Educación nombre común femenino. Barcelona: Octaedro, 2006, cap. 8, p.158-183.

BAUMAN, Z. Amor líquido. Acerca de la fragilidad de los vínculos humanos. México: Fondo de Cultura Económica, 2005.

CONTRERAS, J. Personalizar la relación: aperturas pedagógicas para personalizar la enseñanza. Inédito. 2007.

CONTRERAS, J.; PÉREZ DE LARA, N. (Org.). Investigar la experiencia educativa. Madrid: Morata, 2010.

CORBO, E. Breve ensayo sobre lo posible. In: BAQUERO, R.; DIKER, G.; FRIGERIO, G. (Org.). Las formas de lo escolar. Buenos Aires: Del Estante Editorial, 2003, p. 305-322.

COSENTINO, V. Quién evalúa a quién y por qué. In: LELARIO, A.; COSENTINO, V.; ARMELLINI, G. (Org.). Buenas noticias de la escuela. Madrid: Sabina Editorial, 2010, p.135-145

ELLSWORTH, E. Posiciones en la enseñanza. Diferencia, pedagogía y el poder de la direccionalidad. Madrid: Akal, 2005.

FEITO, R. ¿En qué puede consistir ser un "buen” profesor?". Cuadernos de Pedagogía, Barcelona, n. 332, p. 85-89, febrero-2004.

HERNÁNDEZ, F.; TORT, A. Cambiar la mirada sobre el fracaso escolar desde la relación de los jóvenes con el saber. Revista Iberoamericana de Educación, n. 49/8, p.1-11, julio-2009.

VAN MANEN, M. Investigación educativa y experiencia vivida. Barcelona: Idea Books S.A, 2003.

ZAMBONI, C. Pensar en presencia. Inédito. 2011.

ZAMBRANO, M. Filosofía y Educación. Málaga: Agóra, 2007. 


\section{Notas}

${ }^{1}$ Proyecto de investigación financiado por el Centro de Estudios Andaluces de la Junta de Andalucía (PRY031/11): "Factores pedagógicos que favorecen el éxito escolar en estudiantes de enseñanza postobligatoria”, dirigido por la Dra. Nieves Blanco García (Universidad de Málaga)

2 "La mediación es algo que pone en relación dos cosas que antes no estaban en relación o cuya relación se había roto. Es crear vínculos que son origen de algo nuevo. La mediación originaria es la que hace viable la vida humana, es la creación en dónde cada uno y cada una es pensada en su singularidad irreductible”, Asunción López (2006, p.135)

${ }^{3}$ Puesto que la trayectoria incluye todo el curso de vida escolar, los estudiantes identificaron como referentes significativos tanto a docentes de Primaria como de Secundaria.

${ }^{4}$ Tomamos esta idea de ChiaraZamboni $(2011$, p.5) quien explica que: "los vínculos que trascienden la percepción aun partiendo de ella, son los que dan a cada cual la experiencia de la presencia del otro o de la otra". La presencia pues, "es el fruto de un tejido carnal inconsciente que nos vincula". La presencia del otro, continua la autora, nos modifica y somos pasivos frente a esa modificación, por eso a menudo nos defendemos o resistimos.

${ }^{5}$ Saber y saber transmitir responde a la esencia del ser maestra o ser maestro: enseñar algo a alguien, nos recuerda María Zambrano (2006).

${ }^{6}$ Vita Cosentino (2010) afirma que reducir la relación educativa a una relación paritaria es una ficción, pues no es una relación entre pares sino entre dispares. Y la mediación posible en esta relación, entre dispares, es la autoridad.

${ }^{7}$ Zygmunt Bauman (2005) nos habla de cómo el actual contexto neoliberal penetra con fuerza y "formatea" nuestras prácticas y formas de relación. Nuestro modo de estar con los otros y con el mundo; lo hace de manera que la sustancia y la esencia de la relación se deshace, se liquida. Compartimos gran parte de los análisis que se exponen en este libro, pero aquí la imagen líquida que traemos no es la que este autor pone en juego, más bien al contrario. Nuestra imagen del lugar líquido (hablamos de lugar y no de relación) se mira en la ligereza del amor materno. Un amor en el que la madre da lo mejor de sí a todos sus hijos e hijas, sabiendo que cada quien necesita cosas diferentes y haciendo crecer en sí lo que sabe o percibe como necesario para el otro. Es, entonces, la imagen del lugar que se abre con ligereza a la capacidad de amar. 
* Dolo Molina Galvañ - Profesora Doctora del Dpto. de Didáctica y Organización Escolar, Universidad de Valencia, Valencia, Espanha.

** Nieves Blanco - Profesora del Dpto. de Didáctica y Organización Escolar, Universidad de Málaga, Espanha. Valencia, Espanha.

*** Soledad García - Profesora del Dpto. de Didáctica y Organización Escolar, Universidad de Sevilla, Espanha. Valencia, Espanha.

\section{Correspondência}

Dolo Molina Galvañ - Dpto. de Didáctica y Organización Escolar, Facultat de Magisteri, Universidad de Valencia. Valencia, Espanha.

Despatx P2-015, Av. Tarongers, 4 - 46022, Valencia, Espanha.

E-mail: m.dolores.molina@uv.es

Recebido em 23 de janeiro de 2013

Aprovado em 20 de março de 2013 